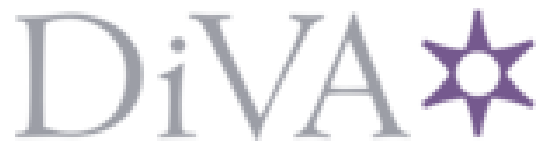

http://www.diva-portal.org

Preprint

This is the submitted version of a paper presented at 11th Nordic Conference on HumanComputer Interaction: Shaping Experiences, Shaping Society (NordiCHI'2O), Tallinn, Estonia, October 25-29, 202O..

Citation for the original published paper:

Reski, N., Alissandrakis, A., Kerren, A. (2020)

Exploration of Time-Oriented Data in Immersive Virtual Reality Using a 3D Radar Chart Approach

In: NordiCHI '2O: Proceedings of the 11th Nordic Conference on Human-Computer Interaction: Shaping Experiences, Shaping Society, Tallinn, Estonia, October 25-29, 2020., 33 (pp. 1-11). New York, NY, USA: Association for Computing Machinery (ACM) https://doi.org/10.1145/3419249.3420171

N.B. When citing this work, cite the original published paper.

Permanent link to this version:

http://urn.kb.se/resolve?urn=urn:nbn:se:lnu:diva-98671 


\section{Exploration of Time-Oriented Data in Immersive Virtual Reality Using a 3D Radar Chart Approach}

NICO RESKI, ARIS ALISSANDRAKIS, VRxAR Labs, Dept. of Computer Science and Media Technology, Linnæus University, Växjö, Sweden

ANDREAS KERREN, ISOVIS, Dept. of Computer Science and Media Technology, Linnæus University, Växjö, Sweden In this paper, we present an approach to interact with time-oriented data in Virtual Reality within the context of Immersive Analytics. We implemented a Virtual Reality application that enables its user to explore data in an immersive environment (head-mounted display, 3D gestural input), utilizing potential advantages of immersive technologies, for instance, depth cues for better spatial understanding, natural interaction, and user engagement. The visualization design is inspired by the overall concept of a radar chart, and using the third dimension to represent time-series related data. We conducted a user study with 15 participants, encouraging them to examine a representative dataset within an explorative analysis scenario with no time constraints. Based on the results of usability and user engagement scores, task completion analysis, observations, and interviews, we were able to empirically validate the approach in general, and gain insights in the users' interaction and data analysis strategies.

CCS Concepts: • Human-centered computing $\rightarrow$ Empirical studies in HCI; Interactive systems and tools; Virtual reality; Gestural input; Empirical studies in visualization; Visualization systems and tools.

Additional Key Words and Phrases: immersive analytics, radar chart, time-oriented data, virtual reality, 3D gestural input

ACM Reference Format:

Nico Reski, Aris Alissandrakis and Andreas Kerren. 2020. Exploration of Time-Oriented Data in Immersive Virtual Reality Using a 3D Radar Chart Approach. In Proceedings of the 11th Nordic Conference on Human-Computer Interaction: Shaping Experiences, Shaping Society (NordiCHI '20), October 25-29, 2020, Tallinn, Estonia. ACM, New York, NY, USA, 18 pages. https://doi.org/10.1145/3419249.3420171

\section{INTRODUCTION}

Recent advances in emerging immersive technologies (e.g., affordable, increased comfort, tracking and detection capabilities) renewed enthusiasm among researchers and developers to create engaging user experiences. Immersive display and interaction technologies, such as Virtual Reality (VR), allow users to dive into computer-generated threedimensional (3D) worlds, exploring them as naturally as one would the real one. While entertainment-related immersive applications are a driving force behind the advances in the consumer market, there are also other, less common, scenarios for such applications, for instance, digital library services [19, 33], space training [31], and architecture prototyping [44]. Another scenario involves the application of immersive technologies for the purpose of supporting data exploration, interaction, and interpretation, as well as analytical meaning- and decision-making. This relatively new field of research is known as Immersive Analytics (IA), and demands cross-disciplinary research expertise from areas such as Information Visualization (InfoVis), Visual Analytics (VA), and Human-Computer Interaction (HCI), to name just a view [14, 16]. Especially under consideration of the Big Data challenge [6], the application of immersive technologies within this

Permission to make digital or hard copies of all or part of this work for personal or classroom use is granted without fee provided that copies are not made or distributed for profit or commercial advantage and that copies bear this notice and the full citation on the first page. Copyrights for components of this work owned by others than the author(s) must be honored. Abstracting with credit is permitted. To copy otherwise, or republish, to post on servers or to redistribute to lists, requires prior specific permission and/or a fee. Request permissions from permissions@acm.org.

(c) 2020 Copyright held by the owner/author(s). Publication rights licensed to ACM.

Manuscript submitted to ACM 
context has the potential to synergize, complement, and enhance overall data exploration and analytics workflows [14], for instance, through utilization of the following:

(1) Immersion can lead to presence, which in return can facilitate user engagement [10].

(2) Intuitive spatial understanding through the visual perception of depth cues (stereopsis, motion parallax) [7, 28].

(3) Visualization of abstract data with a spatial embedding [28].

(4) Decrease of information clutter [7].

(5) Exploration of generally novel and intuitive applications for data visualization and interaction [11, 34, 36].

\subsection{Research Focus}

Our research interest is concerned with the visualization and interaction of multivariate data within an immersive VR setting, utilizing head-mounted display (HMD) devices and 3D gestural input. More specifically, we focus on visualization and interaction of time-oriented data, i.e., all data values in a multivariate dataset are associated with time primitives, thus providing a distinct order of events [2, Ch. 3]. The importance to consider time-related aspects in data exploration is highlighted by Aigner et al. [2, Ch. 1], stating that the majority of data measured today requires relation and contextualization in space and time to be meaningful. Visualization and interaction with time-oriented data remains complex, requiring more research to address challenges such as new visualization methods, novel interaction methods, evaluation, and intertwining visual, interactive, and analytical methods [2, Ch. 8]. Typical tasks within the context of IA are, among others, Encode/Visualize, Select, or Navigate/Explore [10,39]. More specific tasks within the context of time-oriented data are, e.g., tasks related to the examination of the data value's temporal location, rate of change, or synchronization [2, Ch. 4]. It becomes apparent that the design for applications to support such typical task scenarios is a complex endeavour.

In order to address some of these challenges, in this paper we propose an approach to support immersive data analysis and describe the following contributions: (1) development of an interactive application that allows a user to explore time-oriented data in immersive VR; (2) the visualization design is derived from the concept of the radar chart, also known as Kiviat figure [24], and further inspired by the Time-tunnel approach of Akaishi and Okada [3]; (3) using a HMD (HTC Vive) and 3D gestural input (Leap Motion), the user is able to visually observe and interact with the designed visualization for the purpose of explorative data analysis; (4) validation of the overall design in regards to visualization, user interface, and user interaction design, through the conduction of a user interaction study and the measurement of quantitative aspects such as system usability [5, 8], user engagement [30], and task completion, as well as the collection of qualitative data through observations and semi-structured interviews with the participants.

The structure of the paper is such that Section 2 provides an overview of previous work related to IA, radar charts and attempts to transfer these into the 3D domain, and how our approach differs to this related work. Section 3 presents details about the design and functionalities of the implemented VR application. Section 4 describes our evaluation methodology, after which we present the results of the conducted user interaction study in Section 5. Finally, Section 6 concludes this paper by discussing the results and providing impulses for future work.

\section{RELATED WORK}

\subsection{Immersive Analytics}

As a highly interdisciplinary field of research, it is essential to gain introductory knowledge when attempting to design IA experiences, in general [14] as well as under consideration of important physiological fundamentals about VR [25] 
and 3D User Interfaces (3D UIs) [26]. Various interesting research efforts have been conducted recently in order to advance the relatively new domain of IA. For instance, Cordeil et al. [11] describe the development of a toolkit that provides features to interactively author and explore data visualizations in immersive environments, highlighting the importance of data-agnostic and easily accessible tools within this domain. Butcher et al. [9] and Sicat et al. [37] present similar frameworks to support immersive visualization approaches, reporting on their experiences in regards to design, implementation, and evaluation, further indicating a trend towards the need for such prototyping tools. Approaches to visualize and explore data originated on social networks using immersive technologies exist [4, 21, 29], providing insights into real world application use cases and potential benefits, such as supporting user focus and attention, creating affective personal experiences, and facilitating data understanding and representation. The results of a study conducted by Wagner Filho et al. [41] point towards higher usability, higher user preference, and lower mental workload when using immersive display technologies over traditional non-immersive 2D displays in a scenario that featured the exploration of spatio-temporal data in 3D, thus presenting further indications towards the suitability of applying immersive technologies within the context of 3D data visualization. Studies that focus on the investigation of the user experience and interaction design, such as the ones presented by Cordeil et al. [12], Streppel et al. [38], Huang et al. [20], and Reski and Alissandrakis [35], are equally important as they provide recommendations and guidelines in regard to the choice of display and input technologies as well as different interaction techniques in order to actively interact with data in immersive environments. This is particularly relevant for making such IA tools and experiences better suitable to novices and potential end users, who will ideally use these tools frequently.

\subsection{Radar Charts in 3D}

The idea of utilizing a radar chart approach, among others also known as Kiviat figures or star plots, for the purpose of software unit visualization has been described in 1973 by Kolence and Kiviat [23, 24]. Rather than presenting values of individual data variables perpendicular to one another (e.g., histogram or bar chart), they are radially arranged as data variable axes [23]. The values for each data variable along the different adjacent axes can then be connected by a polyline, resulting in a visually interpretable pattern [24] (see visualization within the Information Window as illustrated in Figure 1).

Ever since, the radar chart has become an established method to visualize multivariate data in two dimensions (2D) across various contexts and scenarios. Over years however, with modern 3D graphics computing at hand, various attempts to transfer the concept of the original 2D radar chart into the 3D space have been made, often with the aim to utilize the additional graphical dimension to visualize further information. A common use case is to use that third additional dimension to visually encode time events, visualizing changes in the data over time by stringing together multiple 2D radar charts in 3D. The idea of using a 3D volumetric approach to generate a Kiviat tube within the context of visualizing parallel computing processes has been demonstrated by Hackstadt and Malony [17] and Heath et al. [18]. Akaishi and Okada [3] describe their Time-tunnel approach, a 3D presentation tool viewed through a normal computer monitor, arranging individual time-series data variables in format of 2D line charts as "data-wings" along the third dimension in radial formation. Individual axes can then be rotated and overlapped with other axes in 3D, allowing comparison between different data variables [3]. A series of different 3D axes-based visualization approaches for time-oriented data has also been explored by Tominski et al. [40], for instance as 3D Time Wheel, 3D Multi Comb (similar to Akaishi and Okada [3]), and 3D Kiviat tube (inspired by [17]). A slightly different approach is described by Kerren and Jusufi [22], using the 3D space and a fanning out metaphor to create interactive visualizations of software metrics, allowing users to interact with the individual axes to examine the data in different spatial configurations with the aim 
to overcome occlusion problems). Draper et al. [13] surveyed radial methods within the context of InfoVis, including star plots. Forlines and Wittenburg [15] explored an approach of visualizing radar charts in 3D as Wakame, stacking $2 \mathrm{D}$ radar charts in $3 \mathrm{D}$, creating a hollow tube-like shape that encodes time along the third dimension. The application of temporal radar plots for the purpose of VA has also been explored by Peters [32]. Aiello et al. [1] investigated the placement of individual 2D radar charts in the 3D space with the specific goal to highlight trends in the data variables' values over time. The use of 3D Kiviat plots, similar to the Wakame approach described by Forlines and Wittenburg [15], was extensively explored by Wang [42] in a scenario aimed towards fault detection and process monitoring.

\subsection{Motivation for Radar Charts in Immersive VR}

Approaches of transferring 2D visualizations into the 3D space, as reported in Section 2.2, are promising and certainly invite researchers for further investigations. Particularly in regards to the use of immersive technologies for such analytical purposes (see Sections 1 and 2.1), new and exciting possibilities arise. It is noteworthy that all the work presented throughout Section 2.2 utilized 3D graphics, but were displayed through a non-immersive computer monitor, i.e., a 2D display. With the advantages immersive technologies can provide, for instance better spatial understanding through depth cues (as described in Section 1), revisiting (and potentially reiterating on) 3D visualization and interaction approaches offer oneself naturally for new investigations. Our approach (1) investigates a method of visualizing abstract data inspired by Kiviat figures [24], (2) has similarities to the Time-tunnel approach [3], but (3) allows the user to explore the generated visualization in stereoscopic 3D using VR through HMDs, thus providing the possibility to naturally look at the data by moving the head or simply walking around, while at the same time (4) enabling natural interaction through 3D gestural input where the user can use their hands to interact with the visualization in the immersive VR environment.

\section{IMMERSIVE VR APPLICATION}

We propose an immersive VR application utilizing a HMD and 3D gestural input for visualization and interaction with time-oriented data, specifically based on a 3D radar chart approach. This section describes the VR application's concept and interaction design as well as stating some details about its implementation. ${ }^{1}$

\subsection{Concept and Interaction Design}

On an abstract level, user tasks for the interaction with (time-oriented) data can be divided into three categories: Explorative analysis, confirmative analysis, and presentation of the analysis [2, Ch. 1] [43, Ch. 1]. For the design of our immersive VR application we focus on interaction within the scope of an explorative analysis of data, supporting a scenario of (1) undirected search, where (2) no hypotheses are given, (3) allowing the user to get insights by starting to extract relevant information themselves, in order to (4) come up with own hypotheses [2, Ch. 1]. Interaction with the data is particularly important and useful when exploring unknown data, as it provokes curiosity [2, Ch. 5]. We described typical tasks within this context in Section 1.1.

3.1.1 Visualization in 3D. A visualization of data following our approach in $3 \mathrm{D}$, as previously mentioned similar to the Time-tunnel approach [3], can be described as follows. In 3D, the vertical dimension represents time and is visualized through a black axis with start and end points. With the general concept of radar charts at hand, individual data variables are organized as individual spokes in a radial arrangement around the time axis, i.e., the time-series

\footnotetext{
${ }^{1}$ Video demonstration of the developed IA tool: vimeo.com/393378221.
} 


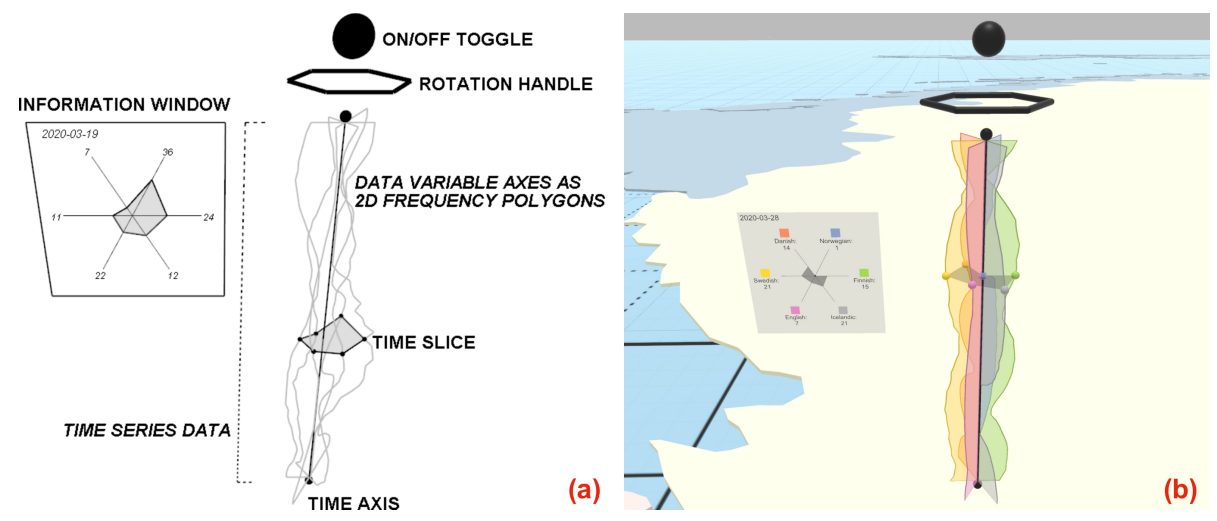

Fig. 1. Concept of the 3D Radar Chart approach. (a) Illustration and (b) implementation. Supplemental $360^{\circ}$ interactive, annotated scenes of the VR application can be viewed online - vrxar.Inu.se/apps/2020-nordichi-3drc/.

data for each variable is visualized as a $2 \mathrm{D}$ frequency polygon. The angular rotation for each spoke is based on the overall amount of different data variables. As the time axis represents the origin for each spoke, naturally if a value (i.e., magnitude or frequency) of a data variable is closer to zero, it is located closer to the time axis, while higher values are farther away. Each data variable spoke is color coded and semitransparent. This arrangement in 3D and the stereoscopic functionalities of the HMD should allow the observer to get a spatial visual impression of the data over time. Rather than creating an occlusive 3D tube, we choose to visualize each data variable spoke as a semitransparent 2D frequency polygon as described above with the aim to avoid occlusion, independent from the observer's viewpoint in 3D. This should allow the observer to visually perceive, at the least, a preview of those data variable spokes that are located behind the ones that are currently in front of the observer, avoiding occlusion. Generally, the intention with the overall design is to provide the observer with the possibility to visually detect patterns, both per individual data variable and in relation to all others. Figure 1 illustrates this concept.

3.1.2 Time Slice as Radar Chart Pattern. In order to examine the data across all data variables for a specific point in time, we display a 2D mesh created from the 3D vertices based on the individual values in all the data variable spokes at that point in time. Consequently, this semitransparent 2D mesh represents the traditional interpretable pattern of a radar chart. Within the context of our application, we call this mesh Time Slice. The main method of navigating forward and backward in time within the 3D visualized data is performed by moving the Time Slice up and down along the (vertical) time axis, which updates the radar chart pattern accordingly. This allows the user to select an individual point in time for further examination. The vertices of the Time Slice are highlighted through spheres, color-coded based on the data variable spokes, to provide further visual guidance. Figure 1a illustrates the presented concept, whereas Figure $1 \mathrm{~b}$ shows the implementation of the Time Slice.

3.1.3 Navigation/Exploration in Time and Information Window. Utilizing 3D gestural input, the Time Slice can be moved twofold. First, the user can reach out with a hand, grab the Time Slice, and drag it up and down along the time axis to the desired point in time by naturally moving up and down the hand (see Figure 2a). This interaction technique can be categorized as direct manipulation through a grasping metaphor [26, Ch. 7]. Second, a two-buttoned graphical menu is attached to the user's left hand palm, enabling stepwise movement forward and backward in time (see Figure 2b). This can be categorized as system control interaction technique [26, Ch. 9]. 

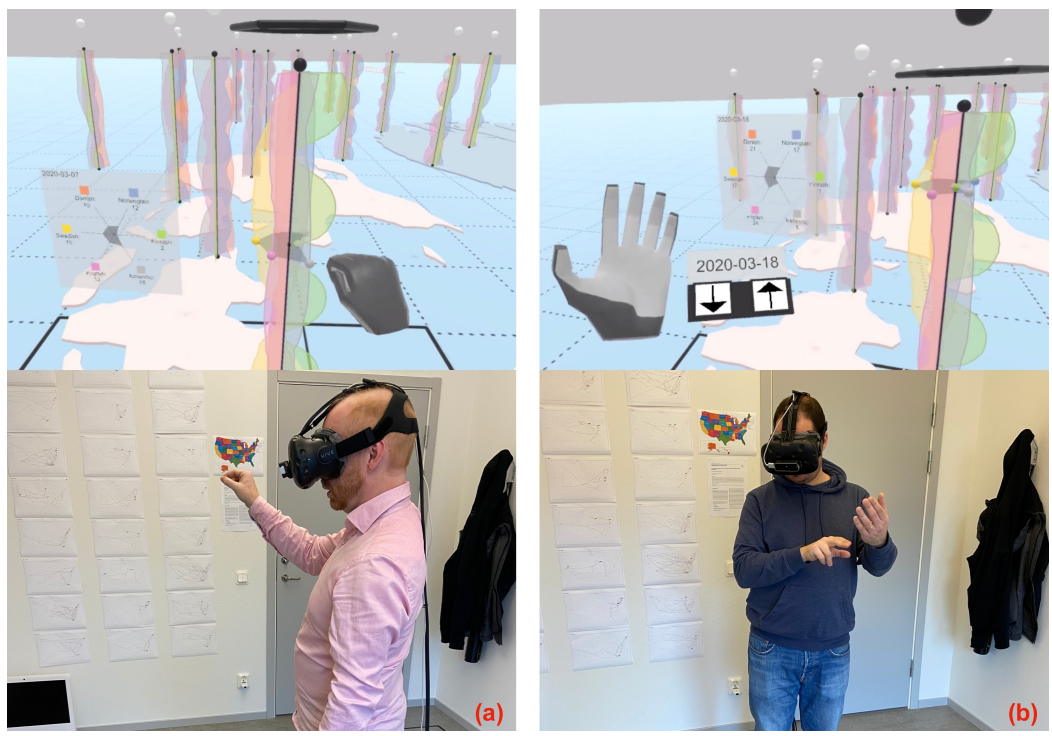

Fig. 2. Exploration in Time. (a) Direct interaction using grabbing, and (b) system-control technique using two-buttoned graphical hand menu. At the bottom: Participants of the user interaction study using the 3D gestural input accordingly.

Furthermore, to provide more details about the data, for instance, an indication about the selected point in time and numerical values for all the data variables, we display a one-panel Information Window next to the 3D visualization. This window provides a more classical 2D visualization as a radar chart for the data at the currently selected point in time, thus corresponding with the position of Time Slice (see Figure 1).

3.1.4 Time Range Selection. We implemented two mechanisms in order to select a specific time range with the aim to support the user with functionalities that allow for a temporally more focused investigation of a period determined as interesting. The first mechanism follows the concept of direct manipulation through a grasping metaphor [26, Ch. 7], enabling the user to perform a pinch hand posture with each of the two hands, and then selecting a desired time range in real-time by moving both hands apart (see Figure 3a). The second mechanism follows the system control technique [26, Ch. 9] by utilizing the Time Slice and a one-button graphical menu attached to the user's right hand palm. The button iterates through three states, the first two of the three being in correspondence with the current position of the Time Slice: (1) Select the start point of the time range selection, (2) select the end point and thus apply the time range selection, and (3) reset to show the entire dataset. Once a start point is selected, a visual highlight provides a preview of the to be selected time range as user feedback (see Figure $3 b$ ).

3.1.5 Additional Interaction Features. It is possible to display multiple 3D radar charts, each representing different data, within the immersive VR environment. For instance, placing charts in different locations in the 3D virtual environment, based on their geospatial properties in the multivariate dataset is arguably a rather natural way of approaching this scenario [4, 27, 35]. Using the room-scale VR setup, the user can then explore the data by walking around. To start interacting with individual 3D radar charts in our application, the user can touch a sphere that is floating above a chart, functioning as a simple on/off toggle. Once turned on, the Time Slice and Information Window are displayed, and logical references between the chart and all the user's graphical hand menus are established. Additionally, a 3D graphical 

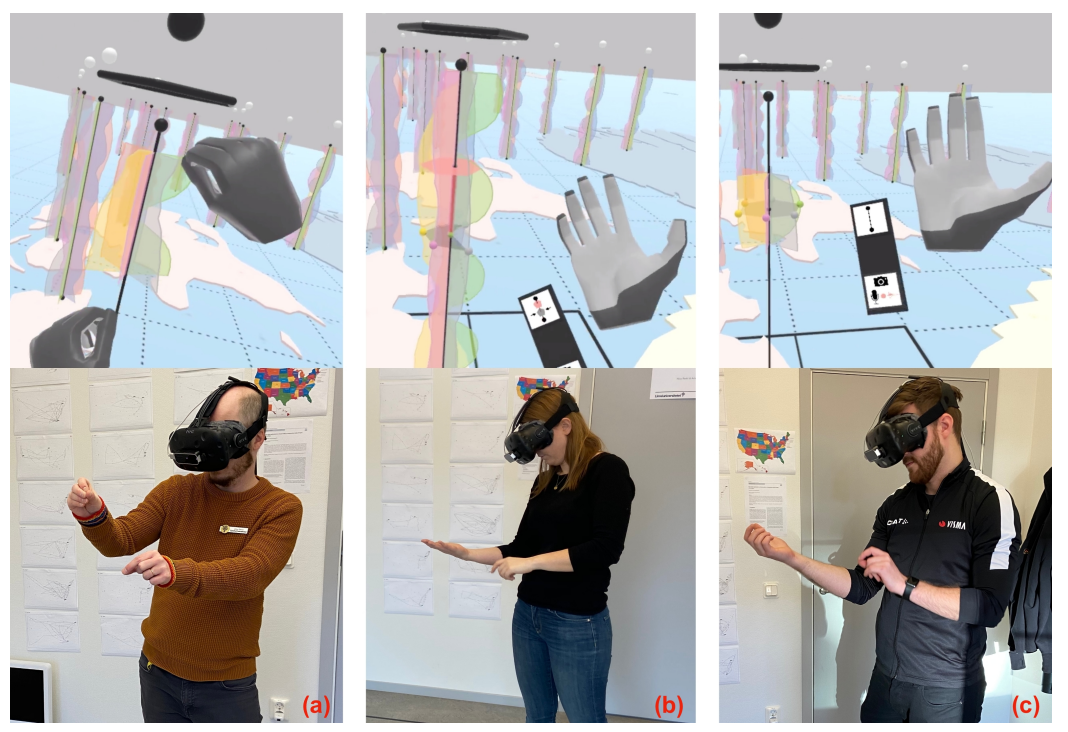

Fig. 3. Interaction with the 3D radar chart. (a) Time range selection using direct interaction by pinching with two hands, (b) time range selection using system-control technique via one-buttoned graphical hand menu, and (c) capturing an annotation using system-control technique via one-buttoned graphical hand menu. At the bottom: Participants of the user interaction study.

rotation handle is displayed above the 3D radar chart, enabling the user to grab and rotate the chart conveniently in place. Furthermore, the vertical placement of the 3D radar chart is automatically calibrated to the the user's height (based on the detected position of the HMD) when the data is loaded, ensuring a similar placement for all users independent of their body height.

3.1.6 Proof-of-concept Feature: Annotation in VR. Finally, as a proof-of-concept feature, we implemented a functionality to capture annotations directly from within the VR application. The main motivation for this feature is derived from the nature of using a HMD: Wearing a HMD isolates oneself from the real world environment, thus making it difficult to write down notes or other annotations alike, which are important for further exploration of the data later on using other, different tools. Therefore it is essential, especially for an IA application, to provide means that allow the analyst to revisit phenomena observed during the immersive exploration activity. At this stage, the implementation of the annotation feature in our application is experimental, and its main aims are to (1) investigate the technical feasibility, and (2) provide a graspable impression of how such a feature could look like, establishing a basis for further discussion and investigation in the future. Following the system control technique [26, Ch. 9], a one-buttoned graphical menu is attached to the user's right hand palm (beneath the time range selection button). Pressing the button triggers two events in iteration: (1) Start an annotation, and (2) end an annotation. While the annotation is ongoing, the user can simply speak aloud noteworthy observations and thoughts, which are recorded by the application using the microphone. Additionally, two screenshots are captured based on the user's view at the start and the end of the annotation. Once an annotation has ended, the recorded audio and image data are transferred to a server. After the immersive exploration activity has finished, the user can play back and view the annotations in format of an illustrative report via normal web browser outside VR. Figure 3c illustrates the graphical hand menu in the VR application to capture annotations. 


\subsection{Implementation}

We utilize a HTC Vive device for the setup of the room-scale VR environment with a play area of 2.2 by 2.2 meters. The 3D gestural input is possible using a Leap Motion controller, attached to the front side of the HMD. The IA tool is implemented using Unity3D. The SteamVR plugin and Unity Assets for Leap Motion Origin Beta provide programming interfaces. The Leap Motion Interaction Engine assisted the practical implementation of the 3D gesture interaction. To encourage other researchers to use our developed IA tool themselves, we provide the source code. ${ }^{2}$

Audio and image data within the scope of the annotation feature (see Section 3.1.6) are captured directly within the Unity3D application. Upon completion of an annotation recording, the captured data are securely transferred via HTTPS using an implemented RESTful API to a Node.js based web server, where it is stored and made accessible. For the purpose of displaying captured annotations in a normal web browser, a web page has been implemented using HTML, CSS, and D3.js (used for DOM manipulation).

\section{EVALUATION METHODOLOGY}

To gain insights about the practical operation of the developed VR application, we conducted a user interaction study with the main aim of validating the proposed visual and interaction design in regards to the user's ability to make analytical assessments. The results of such an evaluation, based on these initial efforts, are important to determine the direction for the VR application's next iteration, providing impulses and directions for further development in the future. This section describes overall details about the user interaction study and data collection methods.

\subsection{User Interaction Study}

Individual study sessions were conducted in a one-on-one scenario between one participant and one researcher at the time. The conduction of one session was aimed to a duration of approximately 45-60 minutes, whereof the participant would spend approximately 25-30 minutes immersed in VR. All study sessions were conducted in our research group lab, which features a designated area for the VR user to move freely without obstacles.

4.1.1 Data Scenario. The overall data context and scenario presented to the participants was inspired by some of our previous work [4,35]. More specifically, we presented the participant with a use case in which they were tasked to investigate language variability on social media in the Nordic region with a focus on time. We created an artificial dataset, comprising of (1) time-series data for a total of 50 events on a per day basis, (2) six language identifiers as data variables (Swedish, Danish, Norwegian, Finnish, Icelandic, and English; thus having a total of six spokes in a 3D radar chart), and (3) frequencies representing the amount of social media posts for each detected language as data variable values for each of the 50 time events. Based on the previous description, for the study task, we focused on two different radar charts, or data nodes, one placed in Denmark and one placed in Sweden inside the VR environment. Using an artificially designed and created dataset allowed for a more accurate task performance assessment, as compared to using more noisy, real world data.

4.1.2 Tasks. The participants were asked to complete a series of tasks, which involved interacting with two data nodes (initially individually, and then together), and state aloud to the researcher in the room either a parameter value, a date (internally corresponding to the time index of a Time Slice), or a period between two dates (time selection). The tasks

${ }^{2}$ Public repository containing the source code of the developed IA tool: github.com/nicoversity/unity_3dradarchart. 
were designed to evaluate if the participants would be able to correctly determine and analyse certain properties of the data.

For the first data node, the participants needed to determine:

T1 the min and max values for all parameters;

T2 the date when all parameters are minimized/maximized simultaneously as much as possible;

T3 the date when Swedish has the highest value and Finish has the lowest value, and vice versa;

T4a a period that contains the most low/high parameter values.

For the second data node, the participants needed to determine:

T4b a period that contains the most low/high parameter values.

Finally, for both data nodes, the participants needed to determine and compare:

T5 a period in each that contains the most low/high parameter values.

After each time period selection in tasks T4a, T4b, and T5, the participants were additionally asked to use the annotation functionality and capture some observations. Each participant was encouraged to freely explore the data using the functionalities provided through the developed VR application, using their own strategy and pace with no time constraints.

4.1.3 Study Procedure. With the aim to validate the overall design and approach of the VR application in practice at this point, no specific user target group was defined, i.e., no specific prior knowledge was required for participation. Each study session followed the same procedure, consisting of four stages: (1) introduction; (2) warm-up; (3) task completion; (4) post-task data collection. Within the introduction, the participant was welcomed by the researcher, informed about the overall procedure of the session, signed a user consent, and completed a brief questionnaire to inquire information about prior VR experiences. Next, within the warm-up stage, the researcher first explained the data scenario and presented the developed VR application to the participant. ${ }^{3}$ After the explanation, the participant was given time to become familiar with the VR application, i.e., wearing the HMD, walking around in the room-scale setting, and operating the application application and its features using the 3D gestural input. ${ }^{4}$ Once the participant felt comfortable and familiar in VR (approximately 5-10 minutes, depending on the participant), the task completion stage started by loading the previously described task dataset (see Section 4.1.1). The participant was asked to complete each task in order, as described in Section 4.1.2 (approximately 20-25 minutes, depending on the participant). Once all tasks were completed, the participant was asked to complete the usability and user engagement questionnaires (see Section 4.2). Afterwards, a short semi-structured interview concluded the study, starting with a brief examination of the participant's captured annotations displayed within a web browser.

\subsection{Data Collection}

A mixture of both quantitative and qualitative methods were used for the data collection. For the task completion, each participant was asked to state aloud their answer, which was written down by the researcher.

To evaluate usability aspects of the developed VR application, we asked participants to self-report using the System Usability Scale (SUS) questionnaire [5, 8]. Furthermore, to gain insights about the participants' engagement during the

\footnotetext{
${ }^{3}$ To aid the explanation, researcher and participant watched together the video mentioned in Footnote 1.

${ }^{4}$ It is noteworthy to state that during this warm-up stage, the participants were presented with a dataset different than the one used for the actual user study tasks. This is important, as is allows the participant to become familiar with operating the prototype, while at the same time avoiding that they transfer insights in the data from the warm-up to the task completion stage.
} 


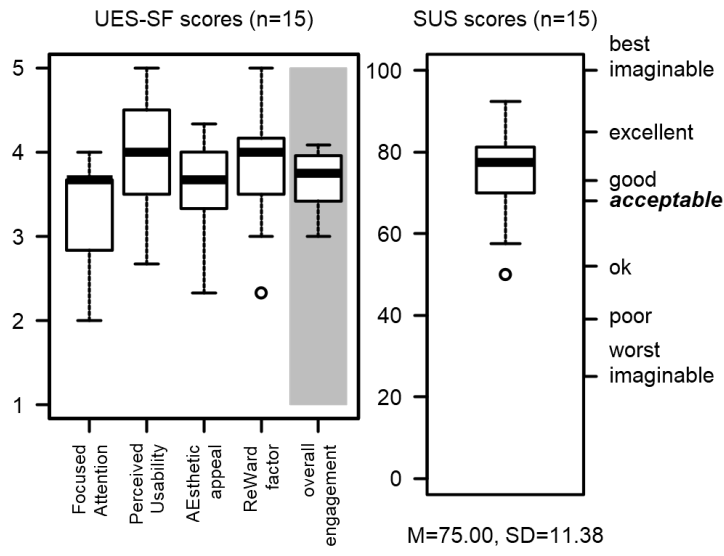

Fig. 4. The UES-SF (left) and SUS (right) scores.

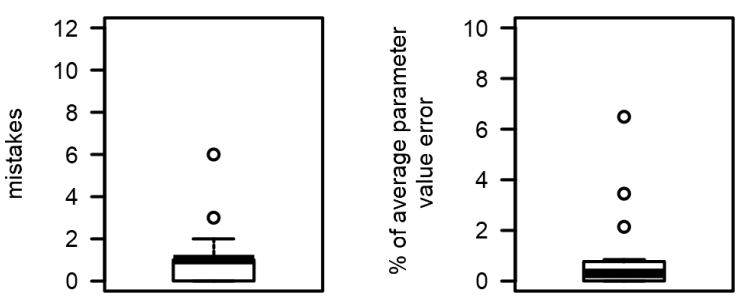

Fig. 5. Determining the min and max values for each of the six parameters, on node one (task T1). Most participants made zero to one mistakes over the twelve questions (left). For those mistakes, the average value error was less than one percent (right).

operation of VR application, we asked them to use the User Engagement Scale - Short Form (UES-SF) questionnaire [30], investigating aspects such as aesthetic appeal, focused attention, perceived usability, and reward. Both questionnaires present individual item statements that are rated using 5-point Likert scale response options, and results are compiled as scores based on each questionnaire's instructions (see Section 5.1).

A short self-constructed questionnaire was used to gain insights in the participants' prior VR experience (see Section 5). The researcher observed the participant during the task completion stage, wrote notes accordingly, and occasionally took photos of the participant for further documentation. Additionally, a brief semi-structured interview with each participant was conducted, inquiring self-assessments about their overall impression of the VR application and its features, as well as revisiting some of the researcher's observations (see Section 5.3).

\section{EVALUATION RESULTS}

A user interaction study with $n=15$ participants was conducted in February / March 2019. Twelve participants categorized their background as technical, one as design related, one as pedagogy related, and one as humanities related. Seven participants reported to have no prior VR experiences, six a little, and two a lot.

\subsection{System Usability Scale (SUS) and User Engagement Scale - Short Form (UES-SF)}

The SUS scores are presented in Figure 4 (right). The mean value was well above the acceptable threshold (i.e., score > 68 , see discussion in [8]). The UES-SF scores are presented in Figure 4 (left); the median values for all factors (including overall engagement) were above average.

\subsection{Task Assessments}

Task T1. Seven participants were able to determine the lowest and highest values for each language parameter, with no mistakes; five made a single mistake (see Figure 5 right). The overall average value error of those mistakes was less than one percent (see Figure 5 left).

Task T2. Figure 6 (right) indicates that although only a few participants managed to determine the exact dates when all parameters were simultaneously minimized or maximized, most were able to come rather close (with two notable 


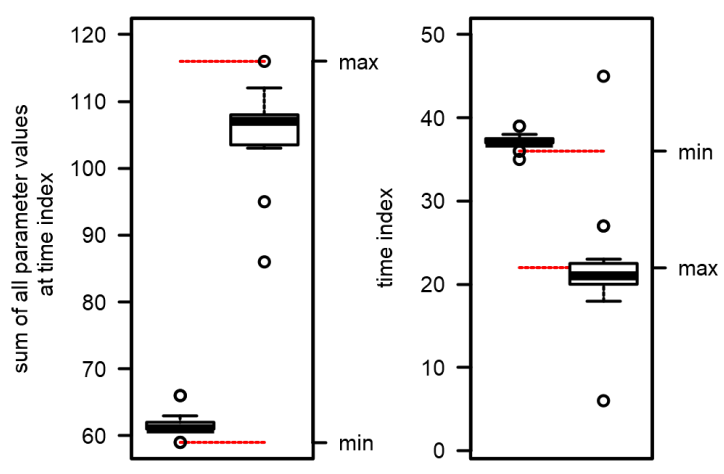

Fig. 6. Determining time indexes when all parameters are minimized or maximized, on node one (task T2). The boxplots on the left show the sum of all parameters at the time index the participants selected, and the boxplots on the right show the time index that the participants selected. For both figures, the correct, target vales for the min and max cases are indicated in red. In respect to both cases (although there were some outliers for the max case), the participants chose a time index very close to the target, which also closely satisfied the task goal.

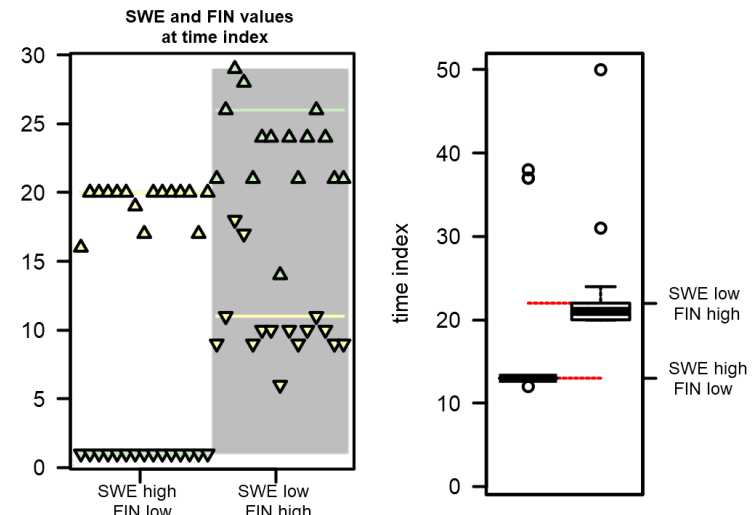

Fig. 7. Determining time indexes when the value for Swedish (SWE) is at its lowest while the value for Finnish (FIN) is at the same time at its highest, and vise versa, on node one (task T3). Except a couple of outliers (that still were close to satisfying the task), most participants chose a time index very close to the predetermined correct answers (right; indicated by red lines). Looking at the parameter values, it was relatively easier to find a close answer for SWE high, FIN low (left).

outliers for the maximized case). Figure 6 (left) indicates that even when not determining the correct date, the sum of the parameter values was appropriately minimized or maximized.

Task T3. It was easier for the participants to determine the date that the Finnish language parameter was at its lowest while the Swedish language parameter was at its highest, given that the lowest Finnish value was the same as the overall minimum; it was more difficult for the vise versa case, as the lowest Swedish value was much higher than the overall minimum (see Figure 7 left). However, Figure 7 (right) shows that for both cases the participants were able to determine a date very close to the correct one.

Tasks T4a and T4b. Given that the participants were not restricted regarding the length of the selected time periods (both in T4a/b and T5), a precise solution could not be pre-determined. However, the individual dates (not periods) where the sum of all parameters is minimized /maximized, and those values, are indicated in all relevant following figures. Figure 8 illustrates the time periods determined initially $(\mathrm{T} 4 \mathrm{a} / \mathrm{b})$ for the two nodes, containing the most low and high parameter values. Some participants selected short periods while others relatively long periods; however some consensus can be seen. Figure 9 shows the mean sum of the parameter values from the selections in Figure 8 , and indicates that despite the variance in the selections themselves, these mean sums satisfy the task instructions (value medians not far from targets, and low value selections were smaller than the high value selections).

Task T5. Comparing the latter time period selections in Figure 10 with the initial selections in Figure 8 shows similar patterns with less variance around the corresponding dates. Similarly, comparing Figure 11 with Figure 9 also shows consistency with overall less variance. 


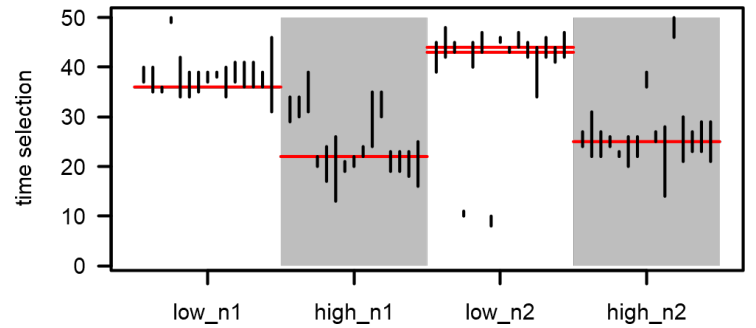

Fig. 8. Initial determination of a time period selection that includes the most low and high values, for both nodes (tasks T4a and T4b). The participants' order is the same to allow comparisons. The red lines indicate the node's min and max time indexes (note that node two has two very close minimums). See also Figure 9.

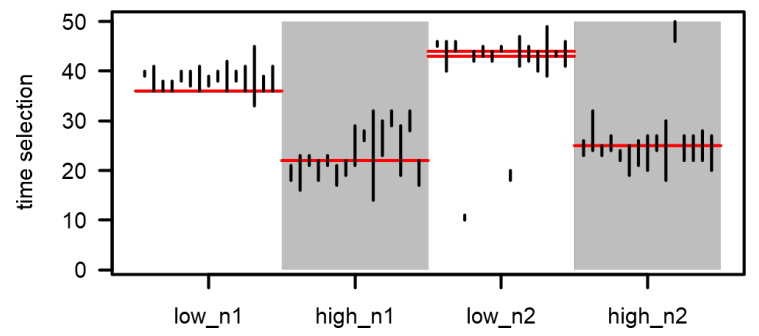

Fig. 10. Later determination of a time period selection that includes the most low and high values, for both nodes (task T5). The participants' order is the same to allow comparisons. The red lines indicate the node's min and max time indexes (note that node two has two very close minimums). See also Figure 11.

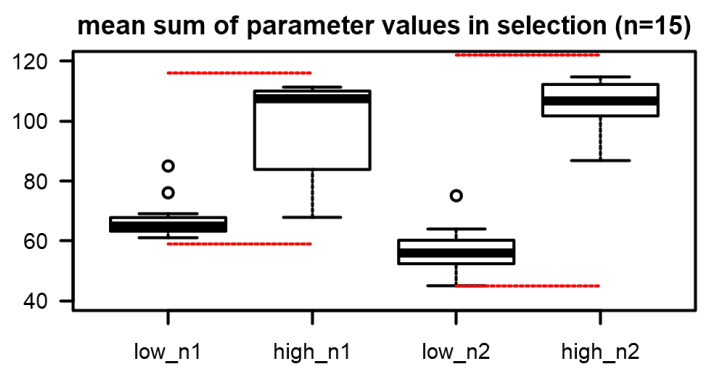

Fig. 9. The mean sum of parameter values for the time period selections shown in Figure 8. The red lines indicate the theoretical possible min and max values for each node.

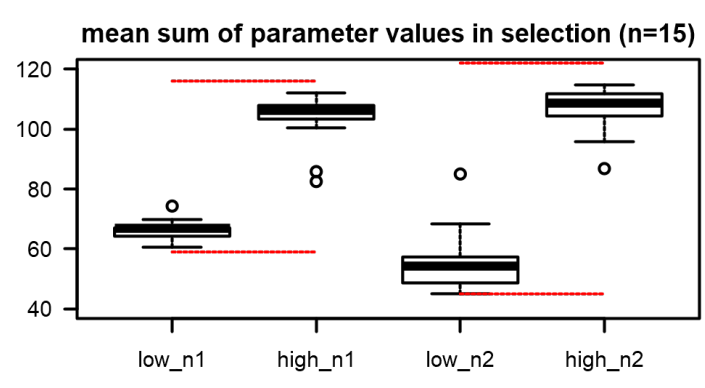

Fig. 11. The mean sum of parameter values for the time period selections shown in Figure 10. The red lines indicate the theoretical possible min and max values for each node.

\subsection{Observations and Semi-Structured Interview}

Notes from the observations during the task completion stage and the semi-structured interviews were combined and generalized into categories representing similar reoccurring themes. They are presented accordingly in the following paragraphs.

Interaction. Eleven participants could be observed to approach the task completion noticeably in a structured and strategic manner, i.e., first generally examining the 3D radar chart by walking around and rotating the chart in place in order to get an overview of the data, and then examining specific points (or periods) in time by strategically moving the Time Slice accordingly to get further detailed information. One participant emphasized the VR application's stereoscopic capabilities, " $3 D$ is actually really good, because you can really see it and get an impression without turning." Another participant positively stated, "It is quite interesting to look at the graph like this. I have this negative impression from [3D UIs in] SciFi movies." Two participants seemed to make not much use of the 3D radar charts time-series visualization overview, but rather focused on moving from time event to time event in order to receive insights from the displayed information window. Eight participants explicitly mentioned that the interaction (using the 3D gestural input) felt "very natural." Comments of the participants included, "I felt like I am already used to it." (first time VR user), "It felt very intuitive, very logically, and easy to learn.", and "Once I learned the pinching, [for the time range selection] I felt fairly 
fluent." Three participants noted that moving the Time Slice sometimes felt "tricky." One participant stated that grabbing and moving the Time Slice felt "a bit uncomfortable over time." In regard to the two provided interaction techniques to select a time range (see Section 3.1.4), six participants were observed mainly using the pinching technique, five mainly used the graphical hand menu technique, and the remaining four used a mixture of both. Participants who preferred the pinching technique elaborated on their choice afterwards with comments such as, "Oh, this [3D gestural input] invites you to do it without buttons.", and "Pinching worked fine and felt more natural than using the hand GUI." A participant who mainly used the graphical hand menu argued, "I preferred the GUI for a more precise selection of the time range."

Annotation. Ten participants were generally enthusiastic and positive towards the demonstration of the implemented annotation feature (see Section 3.1.6) and reviewing the annotations of their own study session in the web browser afterwards, finding it "very useful and meaningful." One participant further highlighted that "Providing an annotation feature is a must for an analysis workflow." Five participants were observed to capture very structured and elaborate annotations (approximately 1-3 minutes in duration per annotation) of their findings within the tasks.

Analytics. One participant explicitly pointed out their ability to visually detect patterns. Other feedback included that the VR application displayed only limited statistics and textual information in terms of numbers (one participant). Furthermore, one participant thought that the scaling along one data variable spoke (i.e., the 2D frequency polygon visualization of the time-series data for each variable) looked sometimes very close to each other, while more detailed examination of the actual data variable values revealed that they were more apart than expected.

Technical. During five study sessions, minor glitches in the sensory tracking of the used VR hardware (HTC Vive) could be observed, occasionally causing the participants to experience brief moments of Vection, i.e., the illusion of self-motion [25, Ch. 8]. The participants stated that it did not impact their experience with the VR application in a major capacity. However, it is noteworthy that one participant asked to take a short (approximately 5 minutes) break between tasks T4a and T4b. One participant was observed to unintentionally apply the time range selection through the pinching interaction technique due to their hand posture detected by the 3D gestural input device (Leap Motion). Furthermore, one participant was observed wanting to naturally interact with objects outside the 3D gestural input device's (Leap Motion) sensory field of view.

\subsection{Limitations}

Some limitations with respect to the results need to be considered. Data was collected from a comparatively modest amount of participants. Therefore, the reported results indicate trends and noteworthy considerations, interpreted within the presented IA context and research focus (see Section 1.1). Additionally, any limitations of the data collection methods (see Section 4.2) are to be taken into account (e.g., subjectivity of the researcher's observations, self-reporting nature inherent of the questionnaire completion through the participants).

\section{SUMMARY AND DISCUSSION}

We set out to investigate the visualization and interaction with time-oriented data in VR within the context of IA. For that purpose, we implemented a VR application that allows its user to examine and interact with data in an immersive environment using HMD and 3D gestural input, enabling them to benefit from potential advantages of immersive technologies (e.g., depth cues for better spatial understanding, natural interaction, user engagement) during the analytical activity. The design of the visualization is informed by the overall concept of a radar chart, and using 
the third dimension to represent time-series related data. We reported on some important fundamentals of IA, and reviewed existing visualization approaches for radar charts in 3D. In a user interaction study, human participants used the implemented VR application to explore data in the use case of investigating language variability on social media in the Nordic region. In a scenario of explorative analysis, the participants were asked to complete a set of tasks, allowing the evaluation of the VR application in regards to usability, user engagement, and task completion. The collected quantitative data as well as additional qualitative data from observation and semi-structured interview notes allowed us to validate the overall approach.

Usability. The reported SUS score points towards a good usability, suggesting that the participants generally accepted the VR application's concept and design, and that it is usable within the presented IA context. Some of the lower SUS scores may be attributed to the observed sensory tracking issues in regards to both the HMD and the 3D gestural input, as well as to some users expressing that the interaction using hands not always worked on the first try. Nevertheless, all participants were able to learn the interaction in a comparatively short amount of time (approximately 5-10 minutes warm-up), with the majority stating that they found the interaction very natural. We believe this is particularly positive, especially due to the fact that most of the participants had either no prior or just a little VR experience before.

User Engagement. The reported UES-SF score points towards an overall above average user engagement, indicating that the participants felt engaged during the operation of the VR application in order to complete the given analytical tasks. This result can be supported through the general enthusiasm the majority of the participants expressed when interacting with the VR application. Most of them engaged in the task completion process quite motivated, approaching the search for a solution in a very strategic manner, making use of the capabilities provided by the immersive technologies as well as the features provided by the developed VR application. Investigating the individual dimensions of the UES-SF questionnaire, it becomes apparent that the reported perceived usability is in line with the results of the SUS. Even though the researcher provided no indications about the participants' performance in regards to their task completion, the majority of participants reported a rewarding experience. Albeit the result for the reported aesthetic appeal can be considered generally okay, it also indicates potential for further improvements, for instance by addressing some of the stated accessibility comments. Furthermore, it is to be considered that the visual user interface design was purposely held minimalistic in this first iteration of the VR application. The participants reported mixed results with respect to the focused attention, which in hindsight may be attributed to the fact that they had to carefully listen to the researcher phrasing the tasks as well as reporting back their results. Arguably, this may have taken away from their experience in the immersive environment.

Task Completion. Given our overall motivation to provide a tool that would support the user with their explorative analysis of data, we are satisfied with the reported task completion results. The results point towards the participants' ability to make use of the 3D radar chart visualization and the provided interaction features in order to complete representative tasks in a satisfying manner, both in regards to the actual completion as well as a rewarding feeling afterwards (see reward factor of UES-SF in Section 5.1). The participants were able to investigate different time events in the data in order to find appropriate solutions, both in regard to individual dates as well as requested time periods. It is noteworthy that the tasks requesting a time period as answer did not specify any duration details, leaving it up to the participant to answer what they thought was most appropriate. While some reported shorter time periods as answers, some reported longer ones. Nevertheless, certain trends in the answers among all participants could be identified, indicating that the majority provided appropriate solutions for the given tasks. 
Interaction. The interactive functionalities enabled through the 3D gestural input were generally perceived positively. Except for minor disruptions caused through technical issues, which are arguably normal and to be expected given the underlying nature and concepts of the applied sensory hardware, the users were able to naturally interact in the immersive environment. The majority of participants enjoyed the direct manipulation interaction technique by grabbing and moving the Time Slice up and down within the 3D radar chart in order to examine different points in time. However, at the same time, it was interesting to observe how some participants made targeted use of the graphical hand menu, i.e., they moved the Time Slice to an area of interest, and then used the two-buttoned hand menu to iteratively move forward and backward in time for further investigation. It seems that they used the general grasping metaphor to move quickly in time, and then used the provided system control technique for a more detailed step-by-step analysis of the time-series data. In regards to the two implemented mechanisms for the time range selection, the participants showed rather mixed preferences. Some really preferred the direct manipulation using pinching with both hands, stating that the 3D gestural input offers itself basically for this. At the same time, some preferred to use the graphical hand menu for the time range selection, arguably because it enabled them to be more precise. Finally, some participants were observed using a mixture of both mechanisms, sometimes trying one, and other times the other. Within the task completion, the researchers did not provide any indications of whether the participant should use one technique over the other, but let it open for the participant to decide. This was entirely exploratory to informally investigate, on the side, a potential preference for one interaction technique over the other. The results do not indicate a clear preference, requiring further investigation in the future. Nevertheless, independent of the used interaction technique, the participants were able making use of the time range selection features to solve their tasks, arguably approving the usability for both of them.

Annotation Feature. The implemented proof-of-concept annotation feature received positive feedback. The participants generally agreed that it is a meaningful addition to such a type of application, and especially useful (and necessary) within the context of IA. It was particularly interesting to observe the different annotation strategies of the participants, with some just briefly stating a to the point annotation, while others recorded very elaborate explanations of their observations including to start hypothesizing why certain phenomena in the data might be as they are. The general characteristics of these elaborate annotations point towards the support of the explorative analysis use case, as motivated and described in Section 3.1.

\subsection{Future Work}

The evaluation results of this first iteration of a VR application to explore time-oriented data using a 3D radar chart approach are promising and encourage further investigations in the future. For instance, based on the feedback and under consideration of the various feature requests from the participants, we are motivated to develop a second iteration of the VR application, for instance to include additional filter, zoom, and comparison capabilities, direct annotation in VR, or the ability to take screenshots independently of audio recordings. A next iteration could then be evaluated in a real world case study with a more specific target group, such as data analysts and researchers related to the chosen data context and scenario. Such an evaluation can provide meaningful insights towards the application of such an IA tool within an applied real world analytics workflow. Furthermore, an investigation into the design and extension of the annotation feature seems promising. Finally, we are interested and motivated to investigate potential collaborative capabilities of interacting with such VR application, for instance by adapting and integrating it into a collaborative IA system we are currently working on. 


\section{ACKNOWLEDGMENTS}

The authors wish to thank Angelos Chatzimparmpas, Kostiantyn Kucher, and Jukka Tyrkkö for initial feedback, as well as the participants of the user interaction study.

\section{REFERENCES}

[1] Rossella Aiello, Gennaro Costagliola, Giancarlo Nota, and Fabrizio Torre. 2015. 3DRC Visualizations to Support the Reconciliation of Diverging Project Views. International fournal of Software and Informatics (IFSI) 9, 3 (2015), 331-354. http://ijsi.alljournals.cn/ch/reader/view_abstract.aspx?file_no=i224

[2] Wolfgang Aigner, Silvia Miksch, Heidrun Schumann, and Christian Tominski. 2011. Visualization of Time-Oriented Data (1st ed.). Springer London. https://doi.org/10.1007/978-0-85729-079-3

[3] Mina Akaishi and Yoshihiro Okada. 2004. Time-tunnel: visual analysis tool for time-series numerical data and its aspects as multimedia presentation tool. In Proceedings of the Eigth International Conference on Information Visualization (IV 2004). Institute of Electrical and Electronics Engineers (IEEE), London, UK, 456-461. https://doi.org/10.1109/IV.2004.1320184

[4] Aris Alissandrakis, Nico Reski, Mikko Laitinen, Jukka Tyrkkö, Jonas Lundberg, and Magnus Levin. 2019. Visualizing rich corpus data using virtual reality. Corpus Approaches into World Englishes and Language Contrasts: Studies in Variation, Contacts and Change in English 20 (2019). http://www.helsinki.fi/varieng/series/volumes/20/alissandrakis_et_al/

[5] Aaron Bangor, Philip Kortum, and James Miller. 2009. Determining What Individual SUS Scores Mean: Adding an Adjective Rating Scale. Journal of Usability Studies 4, 3 (May 2009), 114-123. http://uxpajournal.org/determining-what-individual-sus-scores-mean-adding-an-adjective-rating-scale/

[6] Christine L. Borgmann. 2015. Big Data, Little Data, No Data: Scholarship in the Networked World. The MIT Press.

[7] Doug A. Bowman and Ryan P. McMahan. 2007. Virtual Reality: How Much Immersion Is Enough? Computer 40, 7 (13 Aug. 2007$)$, $36-43$. https://doi.org/10.1109/MC.2007.257

[8] John Brooke. 2013. SUS: A Retrospective. Journal of Usability Studies 8, 2 (Feb. 2013), 29-40. http://uxpajournal.org/sus-a-retrospective/

[9] Peter W. S. Butcher, Nigel W. John, and Panagiotis D. Ritsos. 2019. VRIA - A Framework for Immersive Analytics on the Web. In Extended Abstracts of the 2019 CHI Conference on Human Factors in Computing Systems (CHI EA 2019). Association for Computing Machinery (ACM), Glasgow, Schotland, UK, LBW2615:1-LBW2615:6. https://doi.org/10.1145/3290607.3312798

[10] Wolfgang Büschel, Jian Chen, Raimund Dachselt, Steven Drucker, Tim Dwyer, Carsten Görg, Tobias Isenberg, Andreas Kerren, Chris North, and Wolfgang Stuerzlinger. 2018. Interaction for Immersive Analytics. In Immersive Analytics (first online ed.), Kim Marriott, Falk Schreiber Tim Dwyer, Karsten Klein, Nathalie Henry Riche, Takayuki Itoh, Wolfgang Stuerzlinger, and Bruce H. Thomas (Eds.). Springer, Cham, 221-257. https://doi.org/10.1007/978-3-030-01388-2_4

[11] Maxime Cordeil, Andrew Cunningham, Benjamin Bach, Christophe Hurter, Bruce H. Thomas, Kim Marriott, and Tim Dwyer. 2019. IATK: An Immersive Analytics Toolkit. In Proceedings of the IEEE Conference on Virtual Reality and 3D User Interfaces (VR 2019). Institute of Electrical and Electronics Engineers (IEEE), Osaka, Japan, 200-209. https://doi.org/10.1109/VR.2019.8797978

[12] Maxime Cordeil, Tim Dwyer, Karsten Klein, Bireswar Laha, Kim Marriott, and Bruce H. Thomas. 2017. Immersive Collaborative Analysis of Network Connectivity: CAVE-style or Head-Mounted Display? IEEE Transactions on Visualization and Computer Graphics 23, 1 (1 Jan. 2017), 441-450. https://doi.org/10.1109/TVCG.2016.2599107

[13] Geoffrey M. Draper, Yarden Livnat, and Richard F. Riesenfeld. 2009. A Survey of Radial Methods for Information Visualization. IEEE Transactions on Visualization and Computer Graphics 15, 5 (Sept.-Oct. 2009), 759-776. https://doi.org/10.1109/TVCG.2009.23

[14] Tim Dwyer, Kim Marriott, Tobias Isenberg, Karsten Klein, Nathalie Henry Riche, Flak Schreiber, and Wolfgang Stuerzlinger. 2018. Immersive Analytics: An Introduction. In Immersive Analytics (first online ed.), Kim Marriott, Falk Schreiber, Tim Dwyer, Karsten Klein, Nathalie Henry Riche, Takayuki Itoh, Wolfgang Stuerzlinger, and Bruce H. Thomas (Eds.). Springer, Cham, 1-23. https://doi.org/10.1007/978-3-030-01388-2_1

[15] Clifton Forlines and Kent Wittenburg. 2010. Wakame: Sense Making of Multi-Dimensional Spatial-Temporal Data. In Proceedings of the the International Conference on Advanced Visual Interfaces (AVI 2010). Association for Computing Machinery (ACM), Roma, Italy, 33-40. https: //doi.org/10.1145/1842993.1843000

[16] Richard Hackathorn and Todd Margolis. 2016. Immersive analytics: Building virtual data worlds for collaborative decision support. In 2016 Workshop on Immersive Analytics (IA). Institute of Electrical and Electronics Engineers (IEEE), Greenville, SC, USA, 44-47. https://doi.org/10.1109/IMMERSIVE 2016.7932382

[17] Steven T. Hackstadt and Allen D. Malony. 1995. Visualizing Parallel Programs and Performance. IEEE Computer Graphics and Applications 15, 4 (July 1995), 12-14. https://doi.org/10.1109/38.391482

[18] Michael T. Heath, Allen D. Malony, and Diane T. Rover. 1995. Parallel performance visualization: from practice to theory. IEEE Parallel \& Distributed Technology: Systems \& Applications 3, 4 (1995), 44-60. https://doi.org/10.1109/88.473613

[19] Harri Holappa, Johanna Ylipulli, Sami Rautiainen, Ilya Minyaev, Matti Pouke, and Timo Ojala. 2018. VR Application for Technology Education in a Public Library. In Proceedings of the 17th International Conference on Mobile and Ubiquitous Multimedia (MUM 2018). Association for Computing Machinery (ACM), Cairo, Egypt, 521-527. https://doi.org/10.1145/3282894.3289719

[20] Yi-Jheng Huang, Takanori Fujiwara, Yun-Xuan Lin, Wen-Chieh Lin, and Kwan-Liu Ma. 2017. A Gesture System for Graph Visualization in Virtual Reality Environments. In 2017 IEEE Pacific Visualization Symposium (PacificVis). Institute of Electrical and Electronics Engineers (IEEE), Seoul, Korea, 
41-45. https://doi.org/10.1109/PACIFICVIS.2017.8031577

[21] Alexander Ivanov, Kurtis Danyluk, Christian Jacob, and Wesley Willett. 2019. A Walk Among the Data. IEEE Computer Graphics and Applications 39 (May-June 2019), 19-28. https://doi.org/10.1109/MCG.2019.2898941

[22] Andreas Kerren and Ilir Jusufi. 2009. Novel Visual Representations for Software Metrics Using 3D and Animation. In Software Engineering 2009Workshopband (GI-Edition) (Lecture Notes in Informatics (LNI)), J. Münich and P. Liggesmeyer (Eds.). Gesellschaft für Informatik (GI), Kaiserslautern, Germany, 147-154. http://urn.kb.se/resolve?urn=urn:nbn:se:vxu:diva-4228

[23] Kenneth W. Kolence. 1973. The software empiricist. ACM SIGMETRICS Performance Evaluation Review 2, 2 (June 1973), 31-36. https://doi.org/10. $1145 / 1113644.1113647$

[24] Kenneth W. Kolence and Philip J. Kiviat. 1973. Software Unit Profiles \& Kiviat Figures. ACM SIGMETRICS Performance Evaluation Review 2, 3 (Sept. 1973), 2-12. https://doi.org/10.1145/1041613.1041614

[25] Steven M. LaValle. 2019. Virtual Reality. Online. http://vr.cs.uiuc.edu

[26] Joseph J. LaViola, Jr., Ernst Kruijff, Ryan P. McMahan, Doug Bowman, and Ivan P. Poupyrev. 2017. 3D User Interfaces: Theory and Practice (2nd ed.). Addison-Wesley Professional. https://www.pearson.com/us/higher-education/program/La-Viola-3-D-User-Interfaces-Theory-and-Practice-2ndEdition/PGM101825.html

[27] Richard Lutz. 2014. 3D Visualisation of continuous, multidimensional, meteorological Satellite Data. In Proceedings of the 28th Conference on Environmental Informatics (EnviroInfo 2014). Bibliotheks- und Informationssystem (BIS) Verlag, Carl von Ossietzky Universität Oldenburg, Germany, Kaiserslautern, Germany, 147-154. http://enviroinfo.eu/sites/default/files/pdfs/vol8514/0707.pdf

[28] Kim Marriott, Jian Chen, Marcel Hlawatsch, Takayuki Itoh, Miguel A. Nacenta, Guido Reina, and Wolfgang Stuerzlinger. 2018. Immersive Analytics: Time to Reconsider the Value of 3D for Information Visualisation. In Immersive Analytics (first online ed.), Kim Marriott, Falk Schreiber, Tim Dwyer, Karsten Klein, Nathalie Henry Riche, Takayuki Itoh, Wolfgang Stuerzlinger, and Bruce H. Thomas (Eds.). Springer, Cham, $25-55$. https://doi.org/10.1007/978-3-030-01388-2_2

[29] Andrew Moran, Vijay Gadepally, Matthew Hubbell, and Jeremy Kepner. 2015. Improving Big Data visual analytics with interactive virtual reality. In Proceedings of the 2015 IEEE High Performance Extreme Computing Conference (HPEC 2015). Institute of Electrical and Electronics Engineers (IEEE), Waltham, MA, USA, 5. https://doi.org/10.1109/HPEC.2015.7322473

[30] Heather L. O’Brien, Paul Cairns, and Mark Hall. 2018. A practical approach to measuring user engagement with the refined user engagement scale (UES) and new UES short form. International fournal of Human-Computer Studies 112 (April 2018), 28-39. https://doi.org/10.1016/j.ijhcs.2018.01.004

[31] Manuel Olbrich, Holger Graf, Jens Keil, Rüdiger Gad, Steffen Bamfaste, and Frank Nicolini. 2018. Virtual Reality Based Space Operations - A Study of ESA's Potential for VR Based Training and Simulation. In Virtual, Augmented and Mixed Reality: Interaction, Navigation, Visualization, Embodiment, and Simulation (VAMR 2018) (Lecture Notes in Computer Science (LNCS, volume 10909)), Jessie Y. C. Chen and Gino Fragomeni (Eds.). Springer, Cham, 438-451. https://doi.org/10.1007/978-3-319-91581-4_33

[32] Stefan Peters. 2014. Dynamics of spatially extended phenomena - Visual analytical approach to movements of lightning clusters. Ph.D. Dissertation. Technische Universität München, München, Germany. http://publica.fraunhofer.de/documents/N-419044.html

[33] Matti Pouke, Johanna Ylipulli, Ilya Minyaev, Minna Pakanen, Paula Alavesa, Toni Alatalo, and Timo Ojala. 2018. Virtual Library - Blending Mirror and Fantasy Layers into a VR Interface for a Public Library. In Proceedings of the 17th International Conference on Mobile and Ubiquitous Multimedia (MUM 2018). Association for Computing Machinery (ACM), Cairo, Egypt, 227-231. https://doi.org/10.1145/3282894.3282927

[34] Khairi Reda, Alessandro Febretti, Aaron Knoll, Jillian Aurisano, Jason Leigh, Andrew Johnson, Michael E. Papka, and Mark Hereld. 2013. Visualizing Large, Heterogeneous Data in Hybrid-Reality Environments. IEEE Computer Graphics and Applications 33, 4 (July-Aug. 2013), 38-48. https: //doi.org/10.1109/MCG.2013.37

[35] Nico Reski and Aris Alissandrakis. 2020. Open data exploration in virtual reality: a comparative study of input technology. Virtual Reality 24, 1 (2020), 1-22. https://doi.org/10.1007/s10055-019-00378-w

[36] Jonathan C. Roberts, Panagiotis D. Ritsos, Sriram Karthik Badam, Dominique Brodbeck, Jessie Kennedy, and Niklas Elmqvist. 2014. Visualization beyond the Desktop-the Next Big Thing. IEEE Computer Graphics and Applications 34, 6 (Nov.-Dec. 2014), 26-34. https://doi.org/10.1109/MCG.2014.82

[37] Ronell Sicat, Jiabao Li, JunYoung Choi, Maxime Cordeil, Won-Ki Jeong, Benjamin Bach, and Hanspeter Pfister. 2019. DXR: A Toolkit for Building Immersive Data Visualizations. IEEE Transactions on Visualization and Computer Graphics 25, 1 (21 Oct. 2019), 715-725. https://doi.org/10.1109/ TVCG.2018.2865152

[38] Barbara Streppel, Dorothea Pantförder, and Birgit Vogel-Heuser. 2018. Interaction in Virtual Environments - How to Control the Environment by Using VR-Glasses in the Most Immersive Way. In Virtual, Augmented and Mixed Reality: Interaction, Navigation, Visualization, Embodiment, and Simulation (VAMR 2018) (Lecture Notes in Computer Science (LNCS, volume 10909)), Jessie Y. C. Chen and Gino Fragomeni (Eds.). Springer, Cham, 183-201. https://doi.org/10.1007/978-3-319-91581-4_14

[39] Wolfgang Stuerzlinger, Tim Dwyer, Steven Drucker, Carsten Görg, Chris North, and Gerik Scheuermann. 2018. Immersive Human-Centered Computational Analytics. In Immersive Analytics (first online ed.), Kim Marriott, Falk Schreiber, Tim Dwyer, Karsten Klein, Nathalie Henry Riche, Takayuki Itoh, Wolfgang Stuerzlinger, and Bruce H. Thomas (Eds.). Springer, Cham, 139-163. https://doi.org/10.1007/978-3-030-01388-2_5

[40] Christian Tominski, James Abello, and Heidrun Schumann. 2005. Interactive Poster: 3D Axes-Based Visualizations for Time Series Data. In Poster Compendium of IEEE Symposium on Information Visualization (InfoVis 2005). Institute of Electrical and Electronics Engineers (IEEE), Minneapolis, Minnesota, USA, 49-50. https://vcg.informatik.uni-rostock.de/ ct/pub_files/Tominski05AxesBasedVis3D.pdf 
[41] Jorge A. Wagner Filho, Wolfgang Stuerzlinger, and Luciana Nedel. 2020. Evaluating an Immersive Space-Time Cube Geovisualization for Intuitive Trajectory Data Exploration. IEEE Transactions on Visualization and Computer Graphics 26, 1 (1 Jan. 2020), 514-5224. https://doi.org/10.1109/TVCG. 2019.2934415

[42] Ray Chen Wang. 2017. Geometric fault detection using 3D Kiviat plots and their applications. Ph.D. Dissertation. The University of Texas at Austin, Austin, Texas, USA. http://hdl.handle.net/2152/47439

[43] Matthew O. Ward, Georges Grinstein, and Daniel Keim. 2015. Interactive Data Visualization: Foundations, Techniques, and Applications (2nd ed.). A K Peters/CRC Press. https://www.crcpress.com/Interactive-Data-Visualization-Foundations-Techniques-and-Applications/Ward-GrinsteinKeim/p/book/9781482257373

[44] Katrin Wolf, Markus Funk, Rami Khalil, and Pascal Knierim. 2017. Using virtual reality for prototyping interactive architecture. In Proceedings of the 16th International Conference on Mobile and Ubiquitous Multimedia (MUM 2017). Association for Computing Machinery (ACM), Stuttgart, Germany, 457-464. https://doi.org/10.1145/3152832.3156625 